\title{
A arte da comunicação do conhecimento científico
}

\section{The art of communicating scientific knowledge}

\section{El arte de la comunicación del conocimiento científico}

\section{Camila Rezende Pimentel Ribas', Maria Lúcia Zanetti" , Maria Helena Larcher Caliri"II}

\author{
'Psicóloga. Mestranda em Enfermagem Fundamental pela Escola de Enfermagem de Ribeirão Preto da Universidade de São Paulo (EERP/USP). \\ Bolsista Capes. E-mail: camilar.p.ribas@usp.br. \\ "Enfermeira. Professor Associado do Departamento de Enfermagem Geral e Especializada da EERP/USP. E-mail: zanetti@eerp.usp.br.

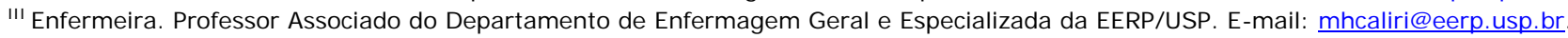

\section{RESUMO}

Reconhecendo que a atividade científica é dependente de uma comunicação eficaz, e que não há ciência sem comunicação, o estudo tem como objetivo descrever e analisar as dificuldades pessoais e situacionais, com base na literatura, relacionadas à publicação de trabalhos científicos em periódicos nacionais e internacionais. Quanto às dificuldades pessoais, identificaram-se a autoconfiança, a motivação e a criatividade em relação à redação do manuscrito e à escolha do veículo mais adequado à divulgação da pesquisa. No que se refere às situacionais, ressaltaram-se a limitação de recursos e a inadequada organização do tempo, entre outros. Assim, a arte da comunicação envolve o desenvolvimento de habilidades pessoais do pesquisador, bem como o manejo das estratégias situacionais. No mundo contemporâneo, a comunicação do conhecimento é fundamental à sintonia entre os canais para a veiculação da informação e as expectativas do atual contexto científico.

Descritores: Publicação; Barreiras de comunicação; Periódico; Conhecimento.

\section{ABSTRACT}

Acknowledging that scientific activity depends on effective communication, and that science does not exist without communication, this study aims to describe and analyze personal and situational difficulties, based on literature, related to the publication of scientific studies in Brazilian and international journals. As to personal difficulties, self-confidence, motivation and creativity were identified with respect to writing the manuscript and choosing the most appropriate journal to disseminate the research. For situational difficulties, limited resources and inadequate time organization stood out, among others. The art of communication involves both developing the researcher's personal skills and managing situational strategies. In the contemporary world, knowledge communication is fundamental for fine-tuning the different channels that publish information with the expectations of the current scientific context.

Descriptors: Publication; Communication barriers; Journal; Knowledge.

\section{RESUMEN}

Reconociendo que la actividad científica depende de una comunicación eficaz, y que no hay ciencia sin comunicación, la finalidad del estudio es describir y analizar las dificultades personales y situacionales, con base en la literatura, relacionadas a la publicación de trabajos científicos en periódicos nacionales e internacionales. Respecto a las dificultades personales, fueron identificadas la autoconfianza, motivación y creatividad con relación a la redacción del manuscrito y la elección del vehículo más adecuado para la divulgación de la investigación. Con relación a las situacionales, se destacaron la limitación de recursos y la organización inadecuada del tiempo, entre otros. Así, el arte de la comunicación involucra al desarrollo de habilidades personales del investigador, y también al manejo de las estrategias situacionales. En el mundo contemporáneo, la comunicación del conocimiento es fundamental para la sintonía entre los canales de diseminación de la información y las expectativas del actual contexto científico.

Descriptores: Publicación; Barreras de comunicación; Periódico; Conocimiento. 


\section{NTRODUÇÃO}

No momento atual de transição entre uma sociedade de informação para uma sociedade do conhecimento, a ciência é um setor estratégico. A produção, transmissão e difusão de informação científica são fundamentais à documentação devido ao seu potencial como fonte de produção de conhecimento e desenvolvimento econômico.

Ao mesmo tempo, o desenvolvimento de tecnologias referentes às Informações na comunicação científica tem contribuído para a globalização da sociedade e para uma suposta nova revolução cultural. A publicação eletrônica responsabiliza-se por promover mudanças quanto à cultura da comunicação científica. As perspectivas advindas pelo acesso livre ao meio eletrônico, à Internet, contribuem para a democratização relativa ao fluxo de informação científica ${ }^{(1)}$.

Nesse contexto da ciência, nada é definitivo e imutável. O domínio dos métodos de pesquisa como instrumentos de busca, análise crítica e síntese dos novos conhecimentos, é fundamental para o crescimento do profissional $^{(2)}$.

Em instituições de ensino e agências financiadoras, a publicação em periódicos internacionais vem sendo valorizada e frequentemente estimada como o fundamental critério no que se refere ao mérito do professorpesquisador, propiciando crescimento relativo à carreira acadêmica, além de favorecer o apoio financeiro de projetos de pesquisa(3).

Nessa vertente, a proposta do presente artigo tem como objetivo descrever e analisar as dificuldades pessoais e situacionais relacionadas à publicação de trabalhos científicos em periódicos nacionais e internacionais, com base na literatura.

\section{Publicação de trabalhos científicos: dificuldades pessoais e situacionais}

Muitos pesquisadores enfrentam dificuldades à publicação de trabalhos em periódicos nacionais e internacionais, e entre essas dificuldades destacamse as pessoais e situacionais.

As dificuldades pessoais implicam em reestruturar ou administrar idéias e sentimentos que funcionam como barreiras à redação dos trabalhos de pesquisa $^{(4)}$. Relacionam-se às perspectivas pessoais e hábitos do trabalho científico; envolvem a necessidade em aumentar a autoconfiança, motivação e criatividade em relação à redação do manuscrito e à elaboração de diretrizes apropriadas para divulgação científica.

Essas dificuldades pessoais relativas à publicação, freqüentemente, interagem com as barreiras situacionais, como por exemplo, sentimentos frustrados acerca da pesquisa, sensação de pouco tempo e redução de disposição e energia para realizar o trabalho necessário. A carência de tempo limita o período disponível para leitura e para a redação de textos, tornando necessárias a organização e a priorização das tarefas mais importantes; direcionando-se, com isso, o autor para a meta de publicação dos trabalhos científicos ${ }^{(4)}$.

Outro fator preponderante e problemático consiste na dificuldade em identificar o veículo mais adequado para divulgar a pesquisa, ou seja, dificuldade em conhecer e utilizar critérios pertinentes à seleção de periódicos para publicação. Além da dificuldade em distinguir apropriadamente se a temática a ser pesquisada é suficientemente relevante ou não para fins de publicação ${ }^{(5)}$.

Ressaltam-se também, dentre os aspectos problemáticos à publicação: a inadequação da amostra e da descrição dos instrumentos, o restrito conteúdo do artigo, a superficialidade de como o objeto de investigação é abordado, a revisão da literatura inapropriada, a pouca originalidade, a ambigüidade da pesquisa e as formulações vagas das hipóteses, entre outros ${ }^{(6)}$.

\section{Desenvolvimento de estratégias para a comunicação do conhecimento científico}

Dentre os aspectos facilitadores no processo de publicação, reconhece-se o valor da metodologia adequada e criteriosa do artigo, a focalização de uma área pouco investigada, além da importância da atualidade, originalidade e relevância do tema a ser abordado, conforme já foi salientado.

O sucesso relativo à comunicação do conhecimento em periódicos nacionais e internacionais envolve, fundamentalmente, a implementação de estratégias viabilizadas para o incentivo, desenvolvimento e expansão de habilidades de pesquisadores quanto à redação do manuscrito e para o enfrentamento das diferentes tarefas que envolvem o processo de publicação.

Vários estudos compartilham a idéia de que a redação de um manuscrito para fins de publicação constitui-se em habilidade aprendida, a qual demanda tempo, instrução e prática(4-5,7). A aprendizagem e o bom desempenho na arte da publicação consistem em um processo contínuo. O exercício dessa prática é fundamental a fim de que o autor possa progressivamente engajar-se na aquisição dessa habilidade.

As habilidades condizentes à redação de estudos para publicação conduzem à objetividade, ao direcionamento às metas, e à capacidade de redigir de maneira clara e organizada.

Outra estratégia no desenvolvimento das habilidades para redação científica consiste na escolha de um mentor intelectual ${ }^{(4,8-10)}$.

Os pesquisadores e autores mais experientes podem atuar como mentores dos neófitos, auxiliando-os na identificação e reflexão de problemas de estudo, incluindo os pesquisadores jovens em seus grupos de pesquisa. Essa atuação visa colaborar na solução de imprevistos e 
emergências relacionados aos problemas dos equipamentos e recursos utilizados na pesquisa, assim como, nos problemas de ordem pessoal, facilitando a comunicação entre pesquisadores acadêmicos de diferentes culturas ${ }^{(7)}$.

Portanto, os mentores têm um papel fundamental na capacitação de novos autores ao propiciar apoio e encorajamento, assim como, idéias, as quais emergem de suas experiências não somente como pesquisadores, mas também como autores, revisores e editores. Nesse sentido, podem compartilhar seus saberes e suas redes de contatos que funcionam como vínculos, contribuindo com os pesquisadores mais jovens ao oferecer uma gama de recursos adicionais que os iniciantes possam vir necessitar ${ }^{(7)}$.

Embora desafiadora, a redação de texto em outro idioma, principalmente na língua inglesa, pode ser apreendida como uma útil estratégia para fins de difusão do conhecimento ${ }^{(2-3)}$. Ao redigir para publicações em revistas internacionais, acultura-se ao processo de publicação e ao idioma da revista.

Existem riscos em relação à utilização de tradutores, devido à falta de domínio dos termos técnicos, específicos, o que pode levar a inadequações referentes à colocação de idéias e termos confusos, ou mesmo errôneos ${ }^{(6)}$.

Acredita-se que há a necessidade de todo pesquisador se apropriar de certo conhecimento relativo ao idioma no qual seu trabalho será publicado, já que através do domínio da língua estrangeira poderá elaborar um texto mais objetivo e adequado, do que possivelmente o profissional tradutor possa vir a realizar. Por outro lado, a revisão do texto por um profissional que tenha o idioma como nativo pode contribuir para maior clareza e adequação do manuscrito.

Outro ponto a ressaltar refere-se à importância de parcerias entre pesquisadores nacionais e internacionais quanto às publicações, em co-autoria.

A co-autoria implica em um modelo colaborativo de apoio ao desenvolvimento de manuscritos para publicação em nível de pós-graduação(11). Co-autores têm suas próprias responsabilidades e devem prestar contas, além de propiciarem contribuições intelectuais.

Quando uma pesquisa é conduzida e publicada, fundamentalmente, estrutura-se uma parceria com colegas; sendo que, nessa associação trabalha-se com a propriedade intelectual, a qual envolve responsabilidades e obrigações de ordem econômica, legal e ética(12)

Quando as instituições acadêmicas e de pesquisa mobilizam a visita de pesquisadores, professores visitantes e especialistas internacionais, fluentes em idiomas estrangeiros, esses podem atuar como facilitadores no desenvolvimento das habilidades do corpo docente, dos alunos e profissionais para a publicação em periódicos internacionais. Mediante o oferecimento de oficinas de trabalho e seminários relativos ao processo de publicação, pode-se contribuir para a capacitação profissional a qual é intrínseca à trajetória dos pesquisadores ${ }^{(6)}$.

Outro aspecto importante refere-se à adequada utilização dos recursos provenientes do meio eletrônico de comunicação (Internet); constituindo-se em importante auxílio para o processo da comunicação do conhecimento científico.

Através desse meio de comunicação faz-se imprescindível uma mente interativa, participativa e aberta à compreensão e ao uso de novas tecnologias em relação à procura de informações relevantes motivadas por nossa curiosidade e, dessa forma, adaptadas, contextualizadas, e por fim, transformadas em conhecimento ${ }^{(13)}$.

Mediante as estratégias que auxiliam na identificação do periódico adequado para veicular a produção científica para a comunidade internacional, constata-se que informações podem ser encontradas em bibliotecas tradicionais e virtuais nos índex ou nos próprios periódicos, além de diversificados sites online ${ }^{(3)}$.

Importa mencionar que a estratégia de disponibilizar os periódicos no formato eletrônico e on-line possibilita uma socialização das comunicações científicas, trazendo benefícios à comunidade acadêmica interessada na produção e utilização do conhecimento.

No entanto, adverte-se que o número elevado de informações disponíveis on-line impõe a necessidade de apuração das informações, na intenção de comprovar a sua validade e adequação; salientandose a importância de tais informações serem complementadas, e jamais substituírem uma discussão com profissionais especialistas ${ }^{(14)}$.

Ultrapassando-se as barreiras pessoais e situacionais, considera-se relevante, também como estratégia, a seleção, criteriosa e pertinente, relativa às ferramentas tanto na busca do referencial bibliográfico como na busca de periódicos científicos de prestígio; sendo tais buscas imprescindíveis à ampliação da possibilidade de aceitação pelo corpo editorial e à garantia da audiência correta.

O prestígio de uma revista relaciona-se à qualidade de arbitragem, ao criterioso processo de avaliação por pares (peer-review); à qualidade dos artigos publicados (originalidade e rigor) e à visibilidade da publicação (alcançada através das indexações em bases de dados e por meio do fator de impacto) ${ }^{(15)}$.

Importa esclarecer que somente revistas indexadas no Institute for Scientific I nformation (ISI) são consideradas para o cálculo do fator de impacto. Esse resulta da divisão do número de vezes em que os artigos de determinada revista são citados em um dado ano, em revistas indexadas pelo ISI, pelo número de trabalhos publicados, nos dois anos anteriores, pelo periódico. 
O fator de impacto influencia a comunidade científica quanto à publicação de seus trabalhos; sendo que a maioria dos pesquisadores, agências financiadoras de pesquisa, instituições de ensino e pós-graduação utilizam o fator de impacto, Science Citation Index ( $\mathrm{SCl})$, da base de dados do ISI divulgado pelo J ournal Citation Reports (JCR) ${ }^{(15)}$.

Em nosso contexto, de inserção na América Latina, o Qualis é o sistema utilizado pela Coordenação de aperfeiçoamento de Pessoal de Ensino Superior (CAPES) para classificação dos periódicos.

Cabe enfatizar que, no processo final e decisivo da escolha de uma revista científica para veicular um artigo é necessário considerar: a qualidade e o momento hábil de revisão, o mérito que a comunidade científica atribui à revista e a contribuição oferecida à sociedade que se apropria da revista(16). Importa, também, se atentar ao risco de se submeter artigos, prematuramente, aos periódicos $^{(17)}$.

\section{Beneficiários das publicações eletrônicas de acesso livre}

De acordo com o avanço tecnológico pertinente ao meio eletrônico de comunicação, a comunicação vem sofrendo importantes transformações. Desde o início da década de 90 , os periódicos eletrônicos de acesso livre começaram a despontar. E, atualmente, as editoras comerciais estão avançando na perspectiva de acesso livre, promovendo a viabilização do acesso para países mais pobres e permitindo o acesso aos artigos seis meses após a primeira publicação(18)

Tanto o pesquisador quanto a editora são beneficiários da política das publicações eletrônicas de acesso livre. Isso pressupõe um ganho ao pesquisador, condizente à facilidade de acesso e conforto.

Já, em relação às editoras, o ganho significa maior fator de impacto devido ao maior número de citações. Ou seja, a evidência de que as citações para textos eletrônicos ocorram em números mais altos (com relação a algumas áreas) que as citações para textos impressos, constituem em motivação para que alguns autores a submetam seus textos à publicação em revistas eletrônicas de acesso livre; o que pode influenciar, de certa forma, as editoras a amenizar seu controle acerca do acesso aos textos que publicam $^{(19)}$.

Assim, presume-se que a publicação eletrônica de livre acesso esteja recebendo um volume significativo de citações, o que é de interesse às editoras, já que as citações elevam prestígio, resultando em mais vendas e conseqüente prestígio.

Recentemente, emergiu uma nova modalidade, via autor, de financiamento do acesso livre, onde ou o autor, ou sua instituição pagam ao editor para que seu artigo venha a ser acessado livremente.
Particularmente, em relação às áreas do conhecimento, em que é rápido o trâmite de renovação, tal inovação vem sendo valorizada(20).

Embora não haja estatísticas, o canal mais difundido de acesso aberto ao conhecimento acadêmico são as páginas individuais utilizadas pelos pesquisadores a fim de divulgação de sua produção pessoal $^{(20)}$. Por fim, percebe-se que a sociedade, como um todo, é beneficiada quando há acesso facilitado à produção científica.

\section{CONSI DERAÇÕES FINAIS}

As dificuldades pessoais e situacionais relacionadas à publicação de trabalhos científicos em periódicos nacionais e internacionais são barreiras possíveis de serem transpostas, com auxílio de estratégias efetivas, além de uma boa dose de motivação perseverante.

Essas dificuldades, também, podem ser flexibilizadas graças à possibilidade do acesso equitativo à informação científica proveniente da publicação eletrônica, contribuindo com espaços de interação e convergência entre usuários, editores e autores; favorecendo, assim, a ampliação do fluxo da comunicação científica.

Sabendo que a comunicação do conhecimento científico abarca fluxo contínuo, tem-se que, gradualmente e progressivamente, quanto maior a experiência do autor no processo de pesquisa, maior será 0 seu desenvolvimento nas habilidades importantes àquilo que pode ser designado como: "arte da publicação". Arte essa, responsável por originar e multiplicar novos conhecimentos, bem como aprimorar metodologias e estratégias.

A agilidade por parte da editoração ainda é fonte de expectativa e angústia dos pesquisadores, embora se compreenda a necessidade dos árbitros assegurarem a qualidade dos manuscritos submetidos à avaliação.

É importante atentar-se ao período ideal para submeter o artigo à publicação e à avaliação de seus pares. Esse processo deve ocorrer no momento em que a pesquisa transmitir uma mensagem convincente, consistente. E, assim, obtido o êxito almejado, ou seja, transformado o texto científico em publicação, a consagração desse valor diferenciar-seá em função do prestígio do periódico em que foi publicado.

A comunicação interliga-se com a disseminação, a qual é interligada à pesquisa, que por fim, interligase com o ensino. Pesquisa-se para o conhecimento daquilo que ainda não se conhece e para a comunicação da descoberta.

Assim, conclui-se que a arte da publicação envolva não apenas a comunicação e disseminação dos resultados da pesquisa, mas também, intrinsecamente, remeta à reflexão de que não há pesquisa sem ensino e não há ensino sem pesquisa. 


\section{REFERÊNCI AS}

1. Castro RCF. Impacto da Internet no fluxo da comunicação científica em saúde. Rev Saude Publica. 2006; 40(esp) : 57-63.

2. Marziale MHP. Produção científica da enfermagem brasileira: a busca pelo impacto internacional. Rev Latino-am Enfermagem. 2005; 13(3):285-6.

3. Caliri MHL, Rocha SMM, Rodrigues ARF. Divulgação da produção científica da enfermagem brasileira em periódicos internacionais. Informativo LatinoAmericano de Enfermagem [Internet] 1999 [cited 2009 set 29];(29):5. Available from: http:// www.eerp.usp.br/ilaenf/29/ilaenf.htm\#mat10.

4. Wills CE. Strategies for managing barriers to the writing process. Nursinbg Fórum. 2000; 35(4): 5-13.

5. Heinrich KT, Neese R, Rogers D, Facente AC. Turn accusations into affirmations: transform nurses into published authors. Nursing Education Perspectives. 2004; 25(3): 139-45.

6. Rassool GH. Writing for international publication in nursing journals: a personal perspective (part 2). Rev Latino-am Enfermagem. 2006; 14(3): 428-34.

7. Montgomery KS, Eddy NL, Jackson E, Nelson E, Reed K, Stark TL, et al. Global research dissemination and utilization. Recommendations for nurses and nurses educators. Nursing and Health Care Perspectives. 2001;22(3): 124-9.

8. Youngblut JM, Brooten D. Institutional research responsibilities and needed infrastructure. J Nurs Scholarsh. 2002; 34(1): 159-164.

9. Baldwin C, Chandler GE. Improving faculty publication output: the role of a writing coach. J Prof Nurs. 2002; 18(1): 8-15.

10. Jacelon CS, Zucker DM, Staccarini JM, Henneman EA. Peer mentoring for tenure-track faculty. J Prof Nurs. 2003; 19(6): 335-8.

11. Van Teijlingen E, Hundley V. Getting your paper to the right journal: a case study of an academic paper. Journal of Advanced Nursing. 2002;37 (6): 506-511.

12. Duncan AM. Authorship, dissemination of research findings and related matters. Appl Nurs Res. 1999; 12(2): 101-6.

13. Caliri MHL. Usando os recursos da Internet na enfermagem. Rev Latino-am Enfermagem. 1997; 5(1): 98-100.

14. Juzzo LMC, Marziale MHP. Critérios para la evaluación de la calidad de las informaciones sobre salud divulgadas online. Nure Investigación. 2005; 14(1): 1-4.

15. Mendes IAC. Enfermagem, conhecimento e comunicação (Editorial). Rev Latino-am Enfermagem. 1996; 4(esp) : 1-2.

16. Bloom FE. The rightness of copyright (Editorial). Science. 1998; 281(5382): 1451.

17. Lawrence PA. The politics of publication. Nature. 2003; 422(6929): 259-61.
18. Wilinsky J. The Nine Flavours of Open Access Scholarly Publishing . J Postgrad Méd. 2003; 49(3): 263-267.

19. Harnad S, Brody T. Comparing the Impact of Open Access (OA) vs. Non-OA Articles in the Same Journals. D-Lib Magazine. [Internet]. 2004 [cited 2009 fev 9];10(6). Available from: http://webdoc.sub.gwdg.de/ edoc/aw/dlib/dlib/june04/ harnad/06harnad.html.

20. Björk BC. Open access to scientific publications an analysis of the barriers to change. Information Research. [Internet]. 2004 [cited 2009 fev 9];9(2):170. Available from: http://InformationR.net/ir/9-2/paper170.html.

Artigo recebido em 03.10.08.

Aprovado para publicação em 13.08.09.

Artigo publicado em 30.09.09. 